\title{
Role of routine hysteroscopy in previous failed ICSI: a continuous clinical dilemma
}

\begin{abstract}
Background: The benefit of hysteroscopy could extend beyond the treatment of intrauterine abnormalities. Irrigation of the cavity with saline may have a beneficial effect on implantation and pregnancy rates, since saline mechanically washes harmful anti-adhesive glycoprotein molecules on the endometrial surface involved in endometrial receptivity, i.e., cyclooxygenase-2 (COX-2), mucin-1 (MUC-1), and integrin.

Objective: The aim of this study is to evaluate the role and benefit of routine hysteroscopy prior to ICSI in women with previous failed ICSI.

Patients and methods: This prospective study has been carried out, at the outpatient clinic, on 100 women that have undergone hysteroscopy as a part of the infertility workup. Women were categorized into three groups according to the number of previous failed IVF/ICSI, once $(n=37)$, twice $(n=33)$, and thrice $(n=30)$.

Results: After analyzing the data, we found that hysteroscopy was associated with 39\% overall clinical pregnancy rate in women with previous/repeat IVF/ICSI failure. Treatment of polyp was associated with $58.3 \%$ clinical pregnancy rate $(N N T=1.71)$. Treatment of intrauterine adhesions was associated with $16.7 \%$ clinical pregnancy rate $(N N T=6)$. Treatment of submucous myoma and uterine septum showed a $75 \%$ and $50 \%$ clinical pregnancy rate, respectively.

Conclusion: Hysteroscopic examination in women with previous failed ICSI may improve pregnancy rate even in the absence of uterine pathology. Consequently, hysteroscopy examination may be proposed as a routine step prior to ICSI in case of previous failure.
\end{abstract}

Keywords: Hysteroscopy, ICSI

\section{Introduction}

Hysteroscopy is a minimally invasive surgical procedure that can view and also operate within the uterine cavity through a transcervical approach. It is a minimally invasive intervention that can diagnose and treat many intrauterine and endocervical problems. Hysteroscopy supplies a profitable expansion for the gynecologist's work enhancing the diagnostic accuracy to allow better management strategies for uterine diseases. After hysteroscopy, the elective surgery could be arranged in a better way (Shinar et al. 2014).

Hysteroscopy could clearly confirm the absence of gross pathology within the uterine cavity and cervical

\footnotetext{
* Correspondence: shssalama@hotmail.com

${ }^{2}$ Department of Reproductive Health \& Family Planning, National Research

Centre, Giza, Egypt

Full list of author information is available at the end of the article
}

canal. It also can differentiate the presence of global pathology from focal lesions. This would be of great benefit in planning which women would require an operative theater, as well as optimizing preoperative surgical planning (Sharma and Yadav 2013).

Hysteroscopy should be recommended in all women where a transvaginal ultrasound showed an abnormally thickened endometrium or suspects an intrauterine pathology. Diagnostic hysteroscopy could be an ordinarily performed gynecological procedure to evaluate the endometrial cavity. There is evidence that hysteroscopy is the gold standard technique for evaluation and diagnosis of any pathology within the uterine cavity. However, hysteroscopy has a limited ability in providing subjective assessment of the depth of myometrial extension of myomas and some other lesions (Shinar et al. 2014). 
Flexible (fiberoptic) hysteroscopes range in diameter from 2.7 to $5 \mathrm{~mm}$ and having a bendable tip that could be deflected in two directions ranging from 120 to $160^{\circ}$. Most also contain an operating channel for tubal catheterization or endometrial biopsy. The flexible hysteroscope is mainly used for office hysteroscopy to easily accommodate within irregularly shaped uterine cavities and move around intrauterine lesions (Shinar et al. 2014).

Rigid hysteroscopes are the most commonly used instruments. Having a broad range of diameters permits for in-office and complex operating-room procedures. Of the narrow options $(3-5 \mathrm{~mm}$ in diameter), the 4-mm telescope (lens) offers the sharpest and clearest view. It accommodates surgical instruments but is small enough to require minimal cervical dilation (Sharma and Yadav 2013).

Numerous studies described the vaginoscopic approach of office hysteroscopy as a well-accepted tolerable procedure that mostly tolerated by the patient without the need of analgesia with a very high success rate of nearly $96 \%$. However, a minor percentage of women may need simultaneous use of local analgesia as cervical block to accomplish the procedure (Firdous et al. 2017).

\section{Patients and methods}

This prospective study has been carried out on 100 women with previous ICSI failure, attended to Ahmed Maher Teaching Hospital, Al-Azhar Teaching Hospital, Medical Research Centre Of Excellence (NRC), and a private IVF center outpatient clinics for infertility workup. Women were categorized according to the number of previous ICSI failures into three research groups once $(n=37)$, twice $(n=33)$, and thrice $(n=30)$. The ethical approval was endorsed by the scientific ethical committee, Faculty of Medicine, $\mathrm{Al}$-Azhar University, and the ethical committee of the National Research Centre.

All women were offered a diagnostic office hysteroscopy as a part of evaluation for women with previous ICSI failure. The purpose of the study was clearly explained to all women, and a printed explanatory consent form was signed and obtained by all study subjects enrolled.

Women included in the study were between 20 and 37 years, BMI $20-30 \mathrm{~kg} / \mathrm{m}^{2}$, nonsmokers for at least 6 months, and with at least one previous ICSI failure. Women with BMI $>30$ or $<20 \mathrm{~kg} / \mathrm{m}^{2}$, > 37 years or $<20$ years, smokers, and medical disorders, i.e., uncontrolled diabetes, hyo/hyperthyroidism, or failed hysteroscopy due to, i.e., cervical stenosis, were excluded from the study.

Diagnostic hysteroscopy was done using usage of a 2.3-mm-diameter continuous flow $0^{\circ}$ grade rigid endoscope (MR-PC, CIRCON ACMI). Optic illumination was provided by a $250-\mathrm{W}$ Xenon light source. Vaginoscopic technique that avoids the need to introduce a speculum and a tenaculum; the vagina, being a cavity, can be distended by introducing the distension medium through the hysteroscope placed into the lower vagina; the anatomy can then be followed by gentle movements of the instrument towards the cervix and cervical canal. Distension of the uterine cavity was performed by using normal saline solution. Normal saline used for uterine distension was instilled from a flexible 500-ml bag wrapped in a pressure cuff connected to a manometer and pumped up to $80-120 \mathrm{~mm} \mathrm{Hg}$. The endometrial surface was inspected systematically, and the tubal ostia were identified. The hysteroscope was then pulled back towards the internal uterine orifice (IUO) to obtain a panoramic view of the whole cavity. The endocervical canal was inspected during withdrawal of the hysteroscope. Uterine abnormal findings such as myomas, polyps, septum, and adhesions were diagnosed and corrected if possible. Completion of the procedure was considered complete only if the entire uterine cavity and both tubal ostia were adequately visualized.

All women were scheduled for ICSI cycle between 1 and 3 months after the hysteroscopy. The ovarian stimulation was done using controlled ovarian stimulation; recombinant FSH was given from the second day of menstrual cycle and continued with adjusting the dose till day of trigger, with gonadotropin-releasing hormone antagonist $250 \mathrm{mg}$ started from day 6 of ovarian stimulation. Trigger was done using HCG $10000 \mathrm{IU}$, and ovum pick up was done 34-37 h later. Two to 3 embryos were transferred into the uterus on days 3 or 5 after pickup. Quantitative $\beta$-HCG estimation was done 14 days after embryo transfer. Clinical pregnancy (defined as women who had sonographic evidence of intrauterine pregnancy with positive fetal cardiac activity) was detected 2 weeks after pregnancy test.

\section{Statistical analysis}

Data were collected, summarized, and tabulated on collection sheets. Data were entered into computer Microsoft Excel sheets with appropriate tabulation and graphical presentation.

Inferential analyses were done for quantitative variables using independent $t$ test in cases of two independent groups, ANOVA test for more than two independent groups with post hoc Tuky's test. In qualitative data, inferential analyses for independent variables were done using chi square test for differences between proportions and Fisher's exact test for variables with small expected numbers. The level of significance was taken at $P$ value $<0.050$ is significant, otherwise is non-significant. 


\section{Results}

In this prospective study, we reviewed 100 women that have undergone hysteroscopy as a part of the infertility workup. Women were categorized into three groups according to the number of previous failed IVF/ICS, once $(n=37)$, twice $(n=33)$, and thrice $(n=30)$.

During the procedure, there were no serious complications observed rather than some pain during insertion (12 women) and difficult visualization in 2 women with polyps due to bleeding (Tables 1, 2, 3, 4, 5, and 6).

\section{Discussion}

Routine hysteroscopy before starting ICSI cycles is still causing a clinical dilemma, with some studies and reproductive centers supporting its routine use, and others would dispute this practice.

Prior research studies revealed and displayed a tendency towards a possible benefit of hysteroscopic screening on ICSI clinical reproductive outcomes. This observation, in conjunction with the detected elevated intrauterine abnormalities prevalence, caused global debate on the value of pre-ICSI/IVF infertility work-up of the endometrial cavity (Khaled et al. 2015).

In the current study, we tried to evaluate the possible role of hysteroscopic examination before IVF/ ICSI performance by studying 100 women with previous failed ICSI.

The unsuspected intrauterine abnormalities prevalence, diagnosed by hysteroscopic examination before ICSI/IVF management, have been estimated to be around $11-45 \%$. Consequently, it have been recommended by various reproductive physicians that these abnormalities have to be diagnosed and managed to enhance the endometrial lining environment and, therefore, the clinical outcome of ICSI management. On the other hand, these recommendations are not supported by high level of research evidence (Smit et al. 2012). Furthermore, the value of hysteroscopic examination in women undergoing first ICSI treatment has not been subjected to adequate research efforts. The European Society for Human Reproduction and Embryology and the Royal College of Obstetricians and Gynecologists do not recommend saline infusion sonohysterography (SIS) nor hysteroscopy as a preliminary investigation before beginning ICSI management (Bahadur et al. 2013).

In our study, we tried to assess the role of hysteroscopy and its possible benefit whether in the presence or absence of uterine pathology. In other words, even in the absence of uterine pathology that needs diagnosis and management via hysteroscopy, still the procedure itself may improve the pregnancy outcome in the next ICSI trial.

In our study population, we recruited 100 women with previous failed ICSI trials (1-3 trials), average age of $30.8 \pm 4.3$ years, and $7.3 \pm 2.3$ years of infertility.

On analyzing our results, 64/100 (64\%) women showed a normal uterine cavity on hysteroscopic examination, 24/100 women had endometrial polyp (24\%), 6/100 women had intrauterine adhesions (6\%), 4/100 women had submucous myoma (4\%), and 2/100 women had uterine septum (2\%).

Furthermore, it was revealed and displayed that the repeat IVF/ICSI cycle clinical outcomes in recruited women in which the no. of retrieved oocytes median $(\mathrm{IQR})=12(7-19)$, fertilization rate median $(\mathrm{IQR})=0.69$ (0.56-0.78), number of transferred embryos 1 embryo in 29 women, 2 embryos in 37 women, and 3 embryos in 34 women.

Additionally, the clinical pregnancy outcome in repeat IVF/ICSI cycle outcomes in recruited women in which 39 women had positive pregnancy test and 61 women had negative pregnancy test (61\%).

Interestingly, the current research study findings revealed and displayed that there were no statistical significant differences between women who had positive clinical pregnancy in repeat IVF/ICSI cycle and women who did not regarding the age, duration of infertility, and no. of previous failed IVF/ICSI cycles ( $p$ values $=$ $0.785,0.586,0.854$, consecutively). Besides that it was shown that there were no statistical significant differences between women who had positive clinical pregnancy in repeat IVF/ICSI cycle and women who did not as regards the no. of retrieved oocytes, fertilization rate and no. of embryos transferred $(p$ values $=0.958,0.583$, 0.293 , consecutively).

Of the recruited 100 study subjects in the current study, hysteroscopy was correlated and linked with an overall clinical pregnancy rate in repeat IVF/ICSI cycle in $39 \%$ of women $(\mathrm{NNT}=2.56)$. Besides, treatment of polyp was associated with $58.3 \%$ clinical pregnancy rate $(\mathrm{NNT}=1.71)$. Treatment of intrauterine adhesions was associated with $16.7 \%$ clinical pregnancy rate $(\mathrm{NNT}=6)$. Treatment of submucous myoma was associated with $75 \%$ clinical pregnancy rate $(\mathrm{NNT}=1.33)$. Treatment of

Table 1 Demographic data of studied women

\begin{tabular}{llll}
\hline & Age (years) & Duration of infertility (years) & No. of previous failed IVF/ICSI trials \\
\hline Range & $24-37$ & $4-11$ & $1-3$ \\
Mean \pm SD or median (IQR) & $30.8 \pm 4.3$ & $7.3 \pm 2.3$ & $2(1-3)$ \\
\hline
\end{tabular}

Data presented as range, mean $\pm \mathrm{SD}$; or range, median (IQR)

$I V F$ in vitro fertilization, ICSI intracytoplasmic sperm injection, $S D$ standard deviation, IQR interquartile range 
Table 2 The association between the no. of previous failed IVF/ICSI trials and hysteroscopic findings

\begin{tabular}{llll}
\hline & Previous failed IVF/ICSI once $(n=37)$ & Previous failed IVF/ICSI twice $(n=33)$ & Previous failed IVF/ICSI thrice $(n=30)$ \\
\hline Normal endometrium & $25(67.6 \%)$ & $18(54.5 \%)$ & $21(70 \%)$ \\
Polyp & $10(27 \%)$ & $9(27.3 \%)$ & $5(16.7 \%)$ \\
Intrauterine adhesions & $2(5.4 \%)$ & $3(9.1 \%)$ & $1(3.3 \%)$ \\
Submucous myoma & $0(0 \%)$ & $2(6.1 \%)$ & $2(6.1 \%)$ \\
Uterine septum & $0(0 \%)$ & $1(3.0 \%)$ & $1(3.3 \%))$ \\
\hline
\end{tabular}

Data presented as number (percentage)

IVF in vitro fertilization, ICSI intracytoplasmic sperm injection

uterine septum was associated with $50 \%$ clinical pregnancy rate $(\mathrm{NNT}=2)$.

As regards the statistical association between no. of previous failed IVF/ICSI trials and hysteroscopic findings, there were no statistically significant association $(p$ value $=0.411$.

Finally, the influence of hysteroscopy and intervention on clinical pregnancy rate in women with previous failed IVF/ICSI trial once, twice, and three times was revealed and displayed in the current research findings. As regards, overall number needed to treat $=2.64,2.54$, and 2.50 , consecutively.

A prior research study similar in methodology to the current research aimed to evaluate the value of usage of the office hysteroscopic examination as a routine investigation in enhancing rates of pregnancy in ICSI in which ICSI was conducted in two research groups of infertile females with no abnormality observed on transvaginal sonographic examination, research group 1: $n=97$ women and research group 2: $n=96$ women, women in research group 1 were subjected to hysteroscopic evaluation before ICSI whereas research group 2 undergone ICSI management without hysteroscopic examination. Then, ICSI was performed for all subjects of the two study groups with no statistically significant difference ( $p$ value $>0.05)$ as regards the number of oocytes retrieved and the number of embryo transfer between research groups. Consecutively, all study subjects have been followed-up for 3 weeks after embryonic transfer for detectability of conception by sonography. The following results were obtained by the research team of investigators in which $43.3 \%$ of research group 1 revealed abnormal hysteroscopic findings. Interestingly, research group 1 revealed a statistically significantly higher pregnancy rate $(70.1 \%)$ in comparison to research group 2 $(45.8 \%)(p$ value $=0.001)$. There was a statistically significant correlation between the usage of hysteroscopy before ICSI and the rate of pregnancy (OR $=2.77,95 \%$ CI [1.53-5.00]). Furthermore, hysteroscopic examination has detected abnormalities in about 50\% of women whose sonography was normal. The research team concluded that routine office hysteroscopic examination is a necessary step for infertility workup prior to ICSI even in women with normal transvaginal sonographic findings. The findings from that research study are in great harmony with the current research findings (Bahadur et al. 2013).

Similarly, another research team have revealed that $21.1 \%$ of women had confirmed abnormalities that needed to be managed before conducting IVF/ICSI and came to the conclusion that routine hysteroscopic examination is crucial before conducting expensive procedures of ART management. On the other hand, contradicting to the current research study findings another research team, came to the conclusion that the value of routine hysteroscopic examination is significant only in women 40 years and older (Smit et al. 2012).

Bohlmann et al. (Bohlmann et al. 2010) research team investigated the prevalence of uterine anomalies in women having recurrent miscarriage. The research team revealed that uterine abnormalities are frequently observed in those women having two and with more than two early miscarriages (36.8-42.9\%).

Table 3 Impact of hysteroscopy and hysteroscopic intervention on clinical pregnancy outcome in repeat IVF/ICSI cycle

\begin{tabular}{llll}
\hline & No. & Positive clinical pregnancy & Number needed to treat (NNT) \\
\hline Overall & 100 & $39(39 \%)$ & 2.56 \\
Normal endometrium & 64 & $20(31.2 \%)$ & 3.20 \\
Polyp & 24 & $14(58.3 \%)$ & 1.71 \\
Intrauterine adhesions & 6 & $1(16.7 \%)$ & 6.00 \\
Submucous myoma & 4 & $3(75 \%)$ & 1.33 \\
Uterine septum & 2 & $1(50 \%)$ & 2.00 \\
\hline
\end{tabular}

Data presented as number (percentage) 
Table 4 The impact of hysteroscopy and intervention on clinical pregnancy rate in women according to number of previous failed IVF/ICSI trials

\begin{tabular}{|c|c|c|c|c|c|c|}
\hline & \multirow{2}{*}{$\begin{array}{l}\text { Previous failed IVF/ICSI once }(n=37) \\
\text { Positive clinical pregnancy }\end{array}$} & \multicolumn{3}{|c|}{ Previous failed IVF/ICSI twice $(n=33)$} & \multicolumn{2}{|c|}{ Previous failed IVF/ICSI thrice $(n=30)$} \\
\hline & & NNT & Positive clinical pregnancy & NNT & Positive clinical pregnancy & NNT \\
\hline Overall & $14(37.8 \%)$ & 2.64 & $13(39.4 \%)$ & 2.54 & $12(40 \%)$ & 2.50 \\
\hline Polyp & $5(50 \%)$ & 2.0 & $5(55.4 \%)$ & 1.80 & $3(60 \%)$ & 1.67 \\
\hline Intrauterine adhesions & $0(0 \%)$ & & $3(100 \%)$ & 1.0 & $0(0 \%)$ & \\
\hline Submucous myoma & - & - & $0(0 \%)$ & - & $2(100 \%)$ & 1.0 \\
\hline Uterine septum & - & - & $1(100 \%)$ & 1.0 & $0(0 \%)$ & - \\
\hline
\end{tabular}

Data presented as number (percentage)

IVF in vitro fertilization, ICSI intracytoplasmic sperm injection, NNT number needed to treat

As regards operative hysteroscopic intervention in infertile women having intrauterine abnormalities the research study of Perez-Medina et al. revealed a rate of clinical pregnancy of $51.4 \%$ in the polypectomy research group after four IUI management cycles, in comparison with $25.4 \%$ in the control research group, corresponding with a number needed to treat to accomplish one additional pregnancy of 3 (NNT $=3$ [95\% CI $=2-5]$. Interestingly, a considerable majority (65\%) of all gestations in the polypectomy research group existed before starting the first IUI management cycle, resulting in a spontaneous conception rate of $29 \%$ in the polypectomy research group versus $3 \%$ in the control research group. These results confirm the hypotheses that intracavitary abnormalities, i.e., endometrial polyps could negatively impact reproductive clinical outcomes (Perez-Medina et al. 2005).

The research study of El-Toukhy et al. which is a multicentric randomized controlled research trial of pre-IVF hysteroscopic interventional assessment in women having more two but less than four IVF trial failures revealed no statistical significant improvement of IVF clinical outcomes in a cohort that have undergone pre-
IVF hysteroscopic examination in comparison with research controls (El-Toukhy et al. 2014).

Finally, a systematic research review and metaanalysis was implemented and evaluate the effectiveness of diagnostic and operative hysteroscopy in enhancing the live birth rate of infertile females, with and without intrauterine anomalies. The primary research outcome assessed was the live birth rate, whereas secondary research outcomes have been rates of pregnancy, rate of miscarriage, and procedurerelated complications. Statistical analytic comparison of hysteroscopy with no hysteroscopy before any (first or consequent) IVF/ICSI trial in infertile women without intrauterine anomalies, there was very lowquality research evidence that hysteroscopic examination raised live birth rate (relative risk $=1.48$, 95\% confidence interval $=1.20-1.81$ ) and moderate quality research evidence that it raised rates of pregnancy $(\mathrm{RR}=1.45,95 \% \mathrm{CI}=1.26-1.67)$. Comparing operative hysteroscopy for intrauterine abnormalities in infertile women with diagnosed polyps or fibroids, there was low-quality research evidence that operative hysteroscopy raises rates of pregnancy $(\mathrm{RR}=2.13,95 \%$

Table 5 Difference between women who had positive clinical pregnancy outcome in repeat IVF/ICSI cycle and women who did not regarding initial characteristics

\begin{tabular}{|c|c|c|c|c|}
\hline & $\begin{array}{l}\text { Positive clinical pregnancy } \\
(n=39)\end{array}$ & $\begin{array}{l}\text { Negative clinical pregnancy } \\
(n=61)\end{array}$ & MD $(95 \% \mathrm{Cl})$ & $P$ \\
\hline \multicolumn{5}{|l|}{ Age (years) } \\
\hline Range & $24-37$ & $24-37$ & 0.24 & $0.785^{\mathrm{a}}$ \\
\hline Mean \pm SD & $30.69 \pm 4.42$ & $30.93 \pm 4.25$ & $(-1.5$ to 2.01$)$ & NS \\
\hline \multicolumn{5}{|c|}{ Duration of infertility (years) } \\
\hline Range & $4-11$ & $4-11$ & -0.25 & $0.586^{\mathrm{a}}$ \\
\hline Mean \pm SD & $7.49 \pm 2.16$ & $7.23 \pm 2.38$ & $(-1.19$ to 0.67$)$ & NS \\
\hline \multicolumn{5}{|c|}{ No. of previous failed IVF/ICSI trials } \\
\hline Range & $1-3$ & $1-3$ & -0.04 & $0.854^{\mathrm{b}}$ \\
\hline Median (IQR) & $2(1-3)$ & $2(1-3)$ & $(-0.37$ to 0.30$)$ & NS \\
\hline
\end{tabular}

Data presented as range, mean $\pm \mathrm{SD}$; or range, median (IQR). $\mathrm{MD}(95 \% \mathrm{Cl})$ : mean difference and its $95 \%$ confidence interval $I V F$ in vitro fertilization, ICSI intracytoplasmic sperm injection, $S D$ standard deviation, IQR interquartile range, NS non-significant ${ }^{a}$ Analysis using independent student's $t$ test

${ }^{\mathrm{b}}$ Analysis using Mann-Whitney's $U$ test 
Table 6 Difference between women who had positive clinical pregnancy outcome in repeat IVF/ICSI cycle and women who did not regarding retrieved oocytes, fertilization rat, and embryos transferred

\begin{tabular}{|c|c|c|c|c|}
\hline & Positive clinical pregnancy $(n=39)$ & Negative clinical pregnancy $(n=61)$ & $\mathrm{MD} / \mathrm{RR}(95 \% \mathrm{Cl})$ & $P$ \\
\hline \multicolumn{5}{|c|}{ No. of retrieved oocytes } \\
\hline Range & $4-25$ & $4-25$ & 0.15 & $0.958^{\mathrm{a}}$ \\
\hline Median (IQR) & $12(8-19)$ & $13(7-20)$ & $(-2.4$ to 2.8$)$ & NS \\
\hline \multicolumn{5}{|l|}{ Fertilization rate } \\
\hline Range & $0.5-0.86$ & $0.5-0.83$ & 0.01 & $0.583^{\mathrm{a}}$ \\
\hline Median (IQR) & $0.7(0.53-0.79)$ & 0.69 (0.57 to 0.73$)$ & $(-0.05$ to 0.04$)$ & NS \\
\hline \multicolumn{5}{|c|}{ No. of embryos transferred } \\
\hline 1 & $14(35.9 \%)$ & $15(24.6 \%)$ & & \\
\hline 2 & $11(28.2 \%)$ & $26(42.6 \%)$ & $1.09^{\mathrm{b}}$ & $0.293^{c}$ \\
\hline 3 & $14(35.9 \%)$ & $20(32.8 \%)$ & (0.79 to 1.49$)$ & NS \\
\hline
\end{tabular}

Data presented as range, mean $\pm \mathrm{SD}$; or range, median (IQR); or number (percentage). $\mathrm{MD}$ (95\% $\mathrm{Cl}$ ) mean difference and its $95 \%$ confidence interval. RR (95\% Cl) risk ratio and its $95 \%$ confidence interval

IVF in vitro fertilization, ICSI intracytoplasmic sperm injection, $S D$ standard deviation, IQR interquartile range, NS non-significant

${ }^{a}$ Analysis using Mann-Whitney's $U$ test

${ }^{\mathrm{b}} \mathrm{RR}$ for ET of 2/3 vs. 1 embryo(s)

${ }^{\mathrm{C}}$ Analysis using chi-squared test

$\mathrm{CI}=1.56-2.92)$. These findings showed support and harmony to the current research study findings (Attilio et al. 2016).

\section{Conclusion}

Hysteroscopic examination had become of remarkable value in current infertility management pathways particularly before ICSI. According to the results of our study, hysteroscopic examination in women with previous failed ICSI may improve pregnancy rate even in the absence of uterine pathology. It may be proposed as a routine step prior to ICSI in women who had previous failures.

However, this recommendation has to be taken with great vigilance due to the limited number of women enrolled in the study. Therefore, larger scale studies are still needed to support or negate our findings.

Also, various endometrial pathologies should be documented among Egyptian women to determine the most common endometrial infertility factors affecting Egyptian women.

\section{Abbreviations}

Cl: Confidence interval; ICSI: Intracytoplasmic sperm injection; IVF: In vitro fertilization; NNT: Number need to treat; RR: Relative risk

\section{Authors' contributions}

EAT contributed to the research idea and study design. FEO conceived the study design and data collection. SaS and SoS contributed to the manuscript writing and data collection. MAR contributed to the data analysis. SA participated in data collection. All authors read and approved the final manuscript.

\section{Funding}

The authors did not receive any financial support from anybody.

\section{Availability of data and materials}

The datasets used and/or analyzed during the current study are available from the corresponding author on reasonable request.

Ethics approval and consent to participate

The ethical approval was endorsed by the scientific ethical committee, Faculty of Medicine, Al-Azhar University, and the ethical committee of the National Research Centre.

\section{Consent for publication}

Written consent was taken from all participants.

\section{Competing interests}

The authors declare that they have no competing interests.

\section{Author details}

${ }^{1}$ Department of Obstetrics \& Gynecology, Faculty of Medicine, Al-Azhar University, Cairo, Egypt. ${ }^{2}$ Department of Reproductive Health \& Family Planning, National Research Centre, Giza, Egypt. ${ }^{3}$ Department of Obstetrics \& Gynecology, Ahmed Maher Teaching Hospital, Cairo, Egypt.

Received: 21 September 2019 Accepted: 6 December 2019 Published online: 19 December 2019

\section{References}

Attilio DS, Costantino DC, Silvia M et al (2016) Efficacy of hysteroscopy in improving reproductive outcomes of infertile couples: a systematic review and meta-analysis. Hum Reprod Update 22(4):479-496

Bahadur A, Malhotra N, Singh N et al (2013) Comparative study on the role of diagnostic hysteroscopy in evaluation of the uterine cavity prior to in vitro fertilization in a developing country. Arch Gynecol Obstet 288(5):1137-1143

Bohlmann MK, von Wolff M, Luedders DW et al (2010) Hysteroscopic findings in women with two and with more than two first-trimester miscarriages are not significantly different. Reprod Biomed Online 21(2):230-236

El-Toukhy T, Khalaf Y, Coomarasamy A et al (2014) A multicenter randomized study of pre-IVF outpatient hysteroscopy in women with recurrent IVF-ET failure- the trophy trial. Hum Reprod 29:36-37

Firdous N, Mukhtar S, Bilal S et al (2017) Role of hysteroscopy and histopathology in evaluating patients with abnormal uterine bleeding. Int J Reprod Contracept Obstet Gynecol 6:615-619

Khaled AAE, Ahmed HA, Mohamed H et al (2015) Routine office hysteroscopy prior to ICSI vs. ICSI alone in patients with normal transvaginal ultrasound: a randomized controlled trial. Arch Gynecol Obstet 291:193-199 
Perez-Medina T, Bajo-Arenas J, Salazar F et al (2005) Endometrial polyps and their implication in the pregnancy rates of patients undergoing intrauterine insemination: a prospective, randomized study. Hum Reprod 20:1632-1635

Sharma JB, Yadav M (2013) New ground breaking International Federation of Gynecology and Obstetrics's classification of abnormal uterine bleeding: Optimizing management of patients. J Midlife Health 4(1):42-45

Shinar S, Bibi G, Barzilay L et al (2014) The value of diagnostic hysteroscopy before operative hysteroscopy for suspected abnormal intrauterine findings. J Minim Invasive Gynecol 21(2):228-232

Smit JG, Jenneke CK, Marinus JC et al (2012) The in SIGHT study: costs and effects of routine hysteroscopy prior to a first IVF treatment cycle. A randomised controlled trial. BMC Womens Health 12:2-6

\section{Publisher's Note}

Springer Nature remains neutral with regard to jurisdictional claims in published maps and institutional affiliations.

\section{Submit your manuscript to a SpringerOpen ${ }^{\odot}$ journal and benefit from:}

- Convenient online submission

- Rigorous peer review

- Open access: articles freely available online

High visibility within the field

- Retaining the copyright to your article

Submit your next manuscript at $\boldsymbol{\nabla}$ springeropen.com 